\title{
Congenital Oligonephronic Renal Hypoplasia with Hypertrophy of Nephrons (Oligonephronia)
}

\author{
K. J. VAN ACKER, H. VINCKE, J. QUATACKER, L. SENESAEL, and J. VAN DEN BRANDE \\ From the Paediatric Clinic and the Department of Pathology of the State University of Gent (Belgium).
}

\begin{abstract}
Van Acker, K. J., Vincke, H., Quatacker, J., Senesael, L., and Van den Brande, J. (1971). Archives of Disease in Childhood, 46, 321. Congenital oligonephronic renal hypoplasia with hypertrophy of nephrons (oligonephronia). Four patients with oligonephronia are described. The clinical picture was that of a progressive renal insufficiency, but the time of onset and severity varied from one case to another. One patient had oligonephronia of one kidney and aplasia of the other kidney. In another patient a familial syndrome of multiple congenital anomalies was observed. In a sib of the latter patient, a simple unilateral renal hypoplasia was discovered.
\end{abstract}

In 1962, Royer et al. described a syndrome which they called congenital bilateral oligonephronic renal hypoplasia with hypertrophy of nephrons ('oligoméganéphronie'). The clinical picture was that of a progressive chronic renal insufficiency, often revealed in early infancy by vomiting, bouts of unexplained fever and dehydration, and later by polyuria and polydipsia and a deficient weight gain. Moderate proteinuria was a constant finding, systemic acidosis, skeletal abnormalities, and renal loss of sodium and chloride were frequently observed. Arterial hypertension and anaemia occurred late. In all cases bilateral hypoplasia of the kidneys was present, in the initial stages characterized by a reduction in the number of nephrons together with a striking increase in the size of these nephrons. After a variable period the renal insufficiency rapidly progressed, death occurring usually after puberty.

The present paper reports the findings in four patients.

\section{Case Reports}

Case 1. P. Rika, a girl, was born at term after a normal pregnancy. Birthweight was $3.0 \mathrm{~kg}$. The parents, not consanguineous, have seven other children. No other cases of nephropathy were known in this family.

At the age of 7 months, polyuria and polydipsia developed in this child, and weight and height gain slowed down. Examination at the age of 13 months showed the clinical, and $x$-ray picture of rickets. A moderate azotaemia was also found. Urine was

Received 23 July 1970 . normal. The patient was treated with conventional doses of vitamin $\mathrm{D}$ and complete healing of the rickets was obtained after 3 months. In a search for the cause of the persisting nitrogen retention and the polyuria, intravenous and retrograde pyelography and cystoscopy were performed at the age of 20 months, which showed a decrease in the size of the right kidney (confirmed later by the criteria of Friedenberg et al., 1965), a normal right urinary tract, and an absence of the left kidney and urinary tract. Long-term treatment with thyroxine was started.

During the next 16 years, the patient was examined at regular intervals. Polyuria and polydipsia were constant findings, except terminally. Apart from a temporary arrest when rickets was present, growth and bone age were always normal. Proteinuria of moderate degree was first detected at the age of $2 \frac{1}{2}$ years and persisted; from the first examination the concentrating capacity was much impaired. Initially, the diluting capacity was normal: it was, however, not examined later. As shown in Fig. 1, the glomerular filtration rate (adjusted for body surface) progressively declined, blood urea and creatinine increasing. Metabolic acidosis was observed from $7 \frac{1}{2}$ years. Terminally, uraemia, hypertension, and severe anaemia developed. Except for the rickets at the age of 13 months, no skeletal abnormalities were observed. An abnormal excretion of red cells and leucocytes was seen only occasionally, concurrently with a urinary infection. A conspicuous urinary loss of sodium and chloride and a hypocalciuria were noted in the course of the last four years. The tubular phosphate reabsorption was decreased throughout. A kidney transplantation was planned and a haemodialysis programme started, when the girl was $17 \frac{3}{4}$, but she died unexpectedly a few weeks later. 


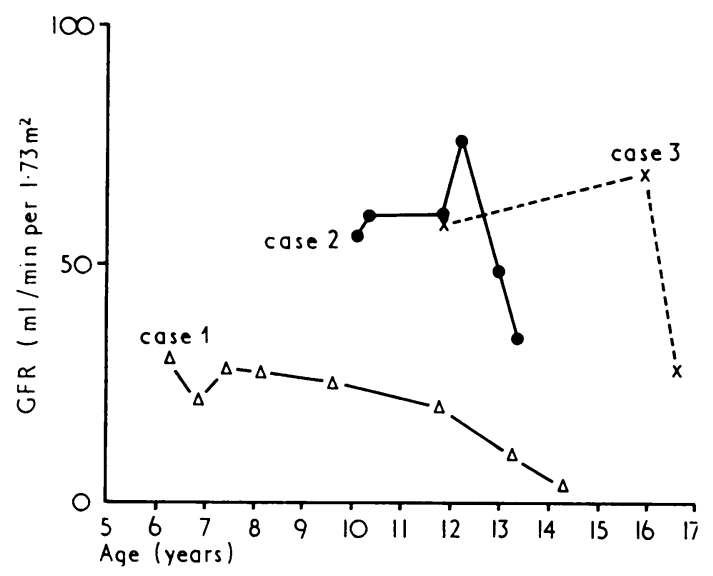

FIG. 1.-Glomerular filtration rate, adjusted for body surface, in Cases 1, 2, and 3.

Case 2. P. Patrick, a boy, was born at term after a normal pregnancy, birthweight $3.5 \mathrm{~kg}$. The parents were not consanguineous; two younger brothers are normal.

The boy was seen for the first time at the age of 3 months as a feeding problem which was ascribed to a proven infection with salmonella. During the next 5 years weight gain and psychomotor development were normal. Urinalysis showed no abnormalities at the age of 10 months and again at $3 \frac{1}{2}$ years.

At the age of $5 \frac{3}{4}$ years, a moderate proteinuria was detected at a routine examination, and was confirmed subsequently. No further examinations were performed until the age of $9 \frac{1}{2}$, when there was a history of polyuria and polydipsia appearing during the past year. On examination, height, weight, and blood pressure were normal. A variable proteinuria, not orthostatic, was present. Blood urea $(85 \mathrm{mg} / 100 \mathrm{ml})$ and creatinine $(1 \cdot 6$ $\mathrm{mg} / 100 \mathrm{ml}$ ) were increased. Concentrating capacity was greatly impaired: urine s.g. never exceeded 1012. Intravenous pyelography suggested symmetrically dwarfed kidneys. Open renal biopsy was carried out at $10 \frac{1}{2}$ years.

The patient is now 14. Polyuria and polydipsia have become severe. Proteinuria has never exceeded $2.5 \mathrm{~g}$ per 24 hours. As shown in Fig. 1, an abrupt fall in glomerular filtration rate occurred after several years. The blood pressure remained normal. Growth and bone age remains normal; there were no skeletal abnormalities. The urinary sediment was always normal and urine cultures remained sterile. Tubular phosphate reabsorption was moderately decreased to normal. The urinary excretion of sodium, chloride, and calcium remained normal.

Case 3. C. Ignace, a boy, was born at term after a normal pregnancy. Birthweight was $4.2 \mathrm{~kg}$. He is the second child of a family of six. There is no consanguinity and the other children are normal.
Proteinuria was discovered on routine examination at the age of 9. Though the boy had never been examined before, history revealed that vomiting had been frequent in the first year, and that polydipsia and polyuria developed later. Psychomotor development was normal. Because of the persisting proteinuria and the increased polydipsia, he was referred at the age of 11 years 10 months. Height and weight were normal. A moderate proteinuria was found, which was not orthostatic. Blood urea $(48 \mathrm{mg} / 100 \mathrm{ml})$ and creatinine $(1 \cdot 1 \mathrm{mg} / 100 \mathrm{ml})$ levels were only slightly increased, but definitely abnormal figures were obtained for inulin clearance $\left(67 \mathrm{ml} / \mathrm{min}\right.$ per $\left.1.73 \mathrm{~m}^{2}\right)$ and PAH clearance (322 $\mathrm{ml} / \mathrm{min}$ per $1 \cdot 73 \mathrm{~m}^{2}$ ). The renal concentrating capacity was much impaired and urine s.g. never exceeded 1009. Diluting capacity was normal. Blood pressure was normal. Intravenous pyelography and renal tomography showed obvious dwarfing of both kidneys. No skeletal abnormalities were seen on $x$-rays, and bone age was normal. A percutaneous renal biopsy was performed.

During the follow-up of more than four years, polydipsia and polyuria increased. Growth remained normal. Glomerular filtration rate is illustrated in Fig. 1. An abrupt fall was noted terminally. Increase in blood urea and creatinine levels was more slowly progressive. Blood pressure remained normal. Urinary infections required regular treatment. Tubular phosphate reabsorption was slightly decreased to normal. Urinary excretion of sodium, chloride, and calcium remained normal. Anaemia developed during the last year, but the general condition of the patient, now 16 years old, is satisfactory.

Case 4. B. Marcel, a boy, was $10 \frac{1}{2}$ years old when admitted for the first time with multiple congenital anomalies. Among the 10 children of this family the following congenital abnormalities were noted: anomalies of the outer ear, the eye, the fingers, and the skeleton, branchial fistulas, dwarfism, otovestibular, neurological and mental disturbances. All these anomalies were present in B. Marcel.

Data for the first 10 years of life are not available: pregnancy was probably uneventful and birthweight was around $3 \mathrm{~kg}$. On examination at the age of $10 \frac{1}{2}$, the psychomotor retardation was obvious. Height and weight were below the 3rd centile, and bone age was much retarded. Proteinuria of less than $1 \mathrm{~g} / 24$ hours was noted. Concentrating capacity was low and could not be corrected by pitressin. Diluting capacity was deficient. Glomerular insufficiency was severe too: creatinine and inulin clearances were respectively 48 and $29 \mathrm{ml} / \mathrm{min}$ per $1 \cdot 73 \mathrm{~m}^{2}$, blood urea was $2 \cdot 3 \mathrm{~g} / 100 \mathrm{ml}$ and blood creatinine $2 \cdot 1 \mathrm{mg} / 100 \mathrm{ml}$. A hyperchloraemic acidosis was present; an acid loading test had to be interrupted because of extreme acidosis. Blood pressure was normal. Urinary sediment was normal. Intravenous and retrograde pyelography, and aortorenography disclosed two very small kidneys, the right kidney being somewhat smaller than the left. The right pelvis was slightly distended but the urinary tract was otherwise 


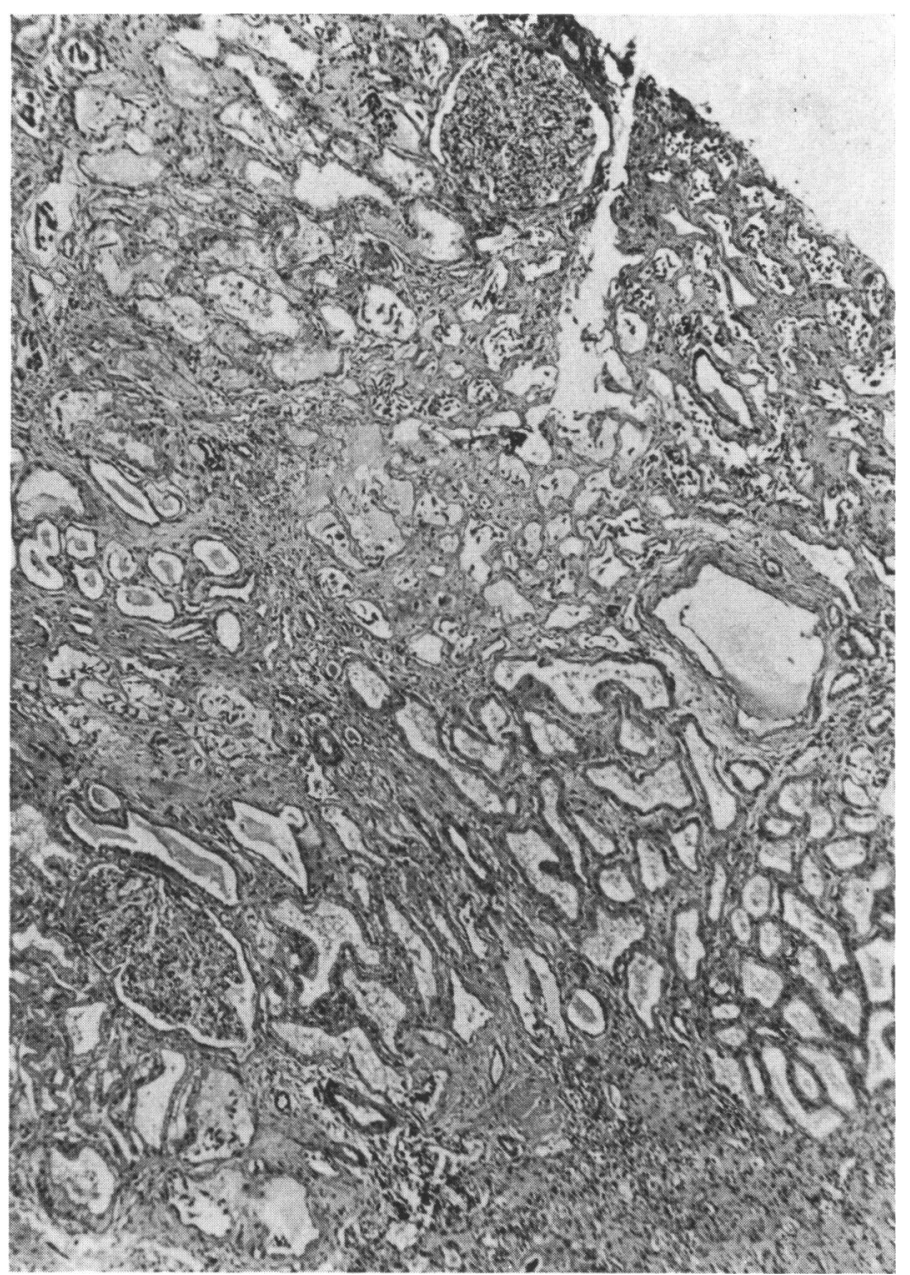

FIG. 2.-(a) Case 1. Kidney, the glomeruli are reduced in number and are abnormally large. (Trichrome. $\times 29$.

normal. The diameter of both renal arteries was much decreased. A generalized aminoaciduria was noted on one occasion. Tubular phosphate reabsorption was decreased. Hypocalciuria and renal loss of sodium and chloride were also noted.

He was readmitted at the age of 12 years 4 months. The findings of the previous observation were confirmed and there was no hypertension. He died elsewhere at the age of $18 \frac{1}{2}$ years; a necropsy was performed by one of us.

\section{Pathologic Data}

Pathological data from two necropsies (Cases 1 and 4) and two biopsies (Cases 2 and 3 ) are available. In Case 1 , the necropsy confirmed the absence of the left kidney; the right kidney weighed $35 \mathrm{~g}$. In Case 4, two small kidneys were found, weighing 22 and $9 \mathrm{~g}$. Due to the hypoplasia of the parenchyma, the left pelvis seemed disproportionately large. In both patients the number of reniculi was reduced to 2 to 4 . The cortex and medulla were difficult to distinguish. On microscopical examination of the necropsy specimens, the glomeruli were strikingly enlarged and their number reduced (Fig. 2a and b). Some glomeruli showed no appreciable abnormalities except for a slight hypertrophy of the capillary epithelial cells. In other glomeruli more or less obvious lesions were present: adhesions between the tuft and the capsule, partial or total hyalinization, and periglomerular fibrosis (Fig. 3). The juxtaglomerular apparatus was moderately enlarged. Some small areas contained no glomeruli at all. Tubules with an increased diameter but with a normal epithelium, dilated tubules with a flattened epithelium, giving rise to a 'thyroid-like picture' (Fig. 4) and atrophic tubules were seen. Most dilated tubules contained granular 


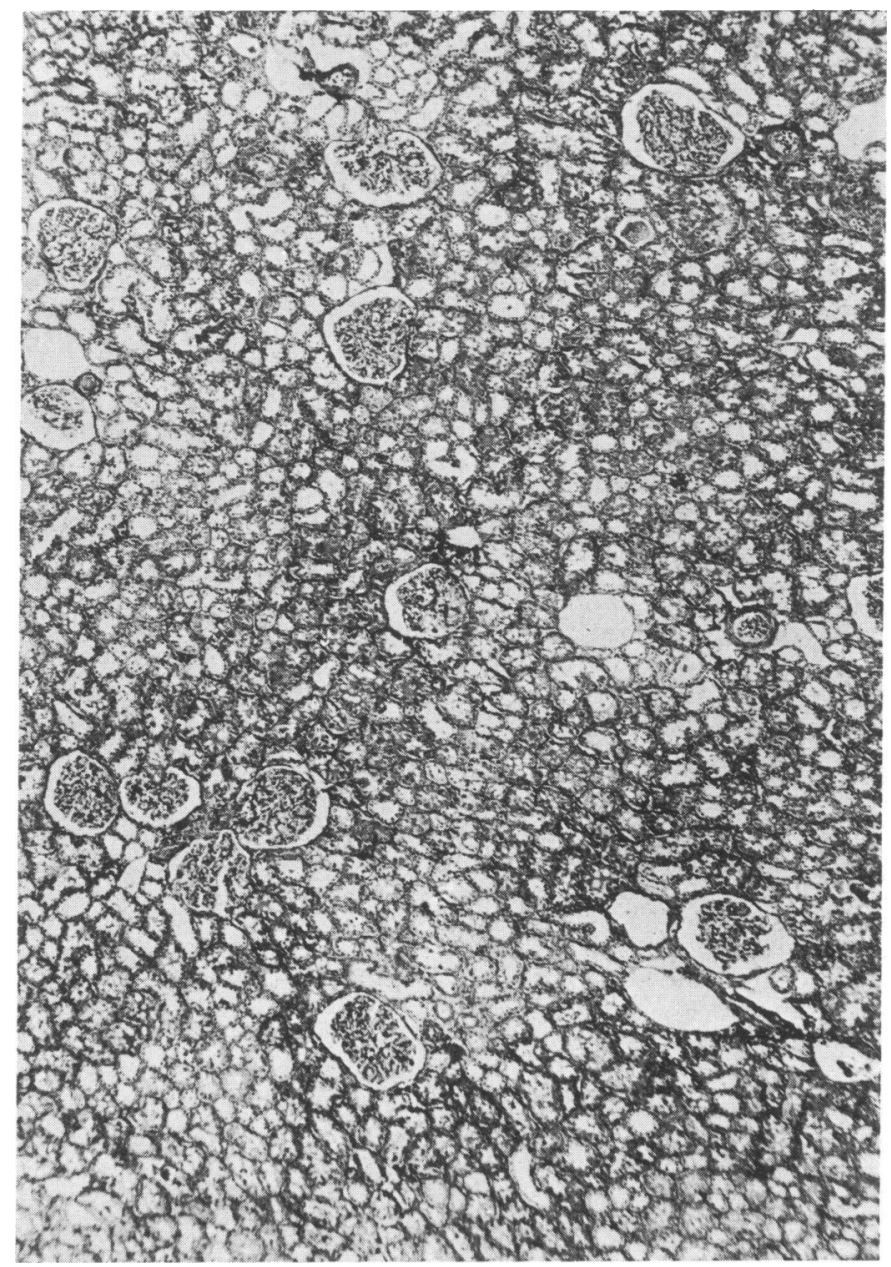

FIG. 2.-(b) Normal kidney from patient of similar age. (Trichrome $\times 29$.)

material and cellular débris. Cellular infiltration and fibrosis of the interstitium were obvious. The walls of the blood vessels were also thickened. In Case 1, the tubular basement membrane was much enlarged. Dysplastic elements were never observed.

The biopsies were performed at the age of $10 \frac{1}{4}$ years (Case 2) and 11 years 10 months (Case 3); as in the necropsy material, the reduced number and the hypertrophy of the glomeruli were the most striking features, when the biopsies were compared with those of other children of the same age group. Practically normal, partially hyalinized, and sclerosed glomeruli were all to be seen. Enlargement of the juxtaglomerular apparatus was very obvious in Case 2. Tubular abnormalities were restricted to dilatation, the tubular epithelium being normal. There was no cellular infiltration, fibrosis of the interstitium, or thickening of the walls of the blood vessels.

\section{The Family of Case 4}

We had the opportunity to examine all the children from the family of Case 4 . In a younger sister of the patient with oligonephronia, a marked hypoplasia of $\frac{D}{O}$ the left kidney was discovered by intravenous pyelography, a finding confirmed by renal scanning, the $N$ urinary tract being otherwise normal. Clinical examination, including weight and length, was normal. Urin- N alysis was also normal except for a sterile pyuria. స్ Blood urea and creatinine, glomerular filtration rate, con- $\sigma$ centrating capacity, and blood pressure were normal. A renal biopsy from the left hypoplastic kidney contained only five glomeruli: the size and the structure of these glomeruli were normal, as were tubules and interstitium.

\section{Discussion}

The findings in these four patients throw some 


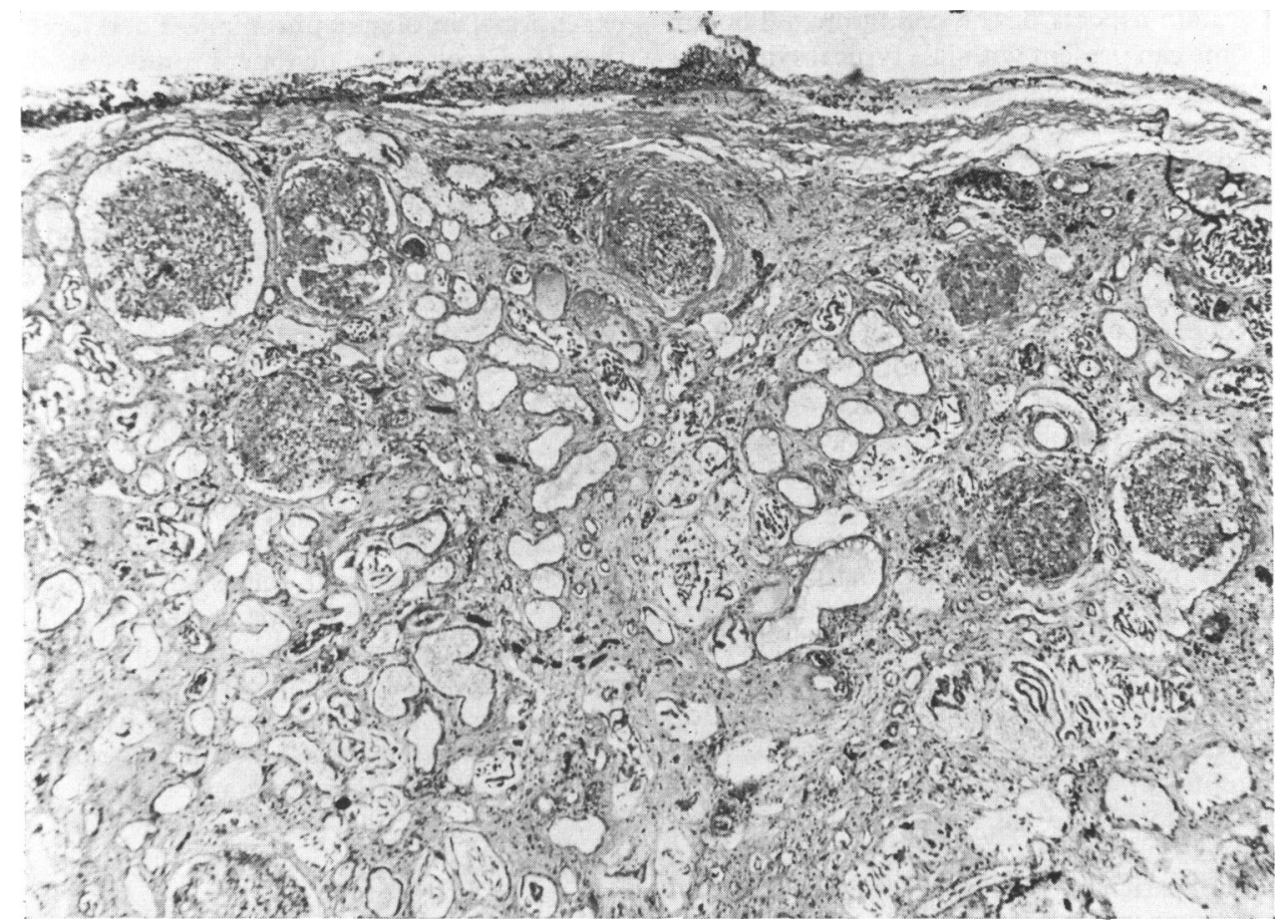

FIG. 3.-Case 4. Glomeruli showing different degrees of damage. (Trichrome. $\times 45$.

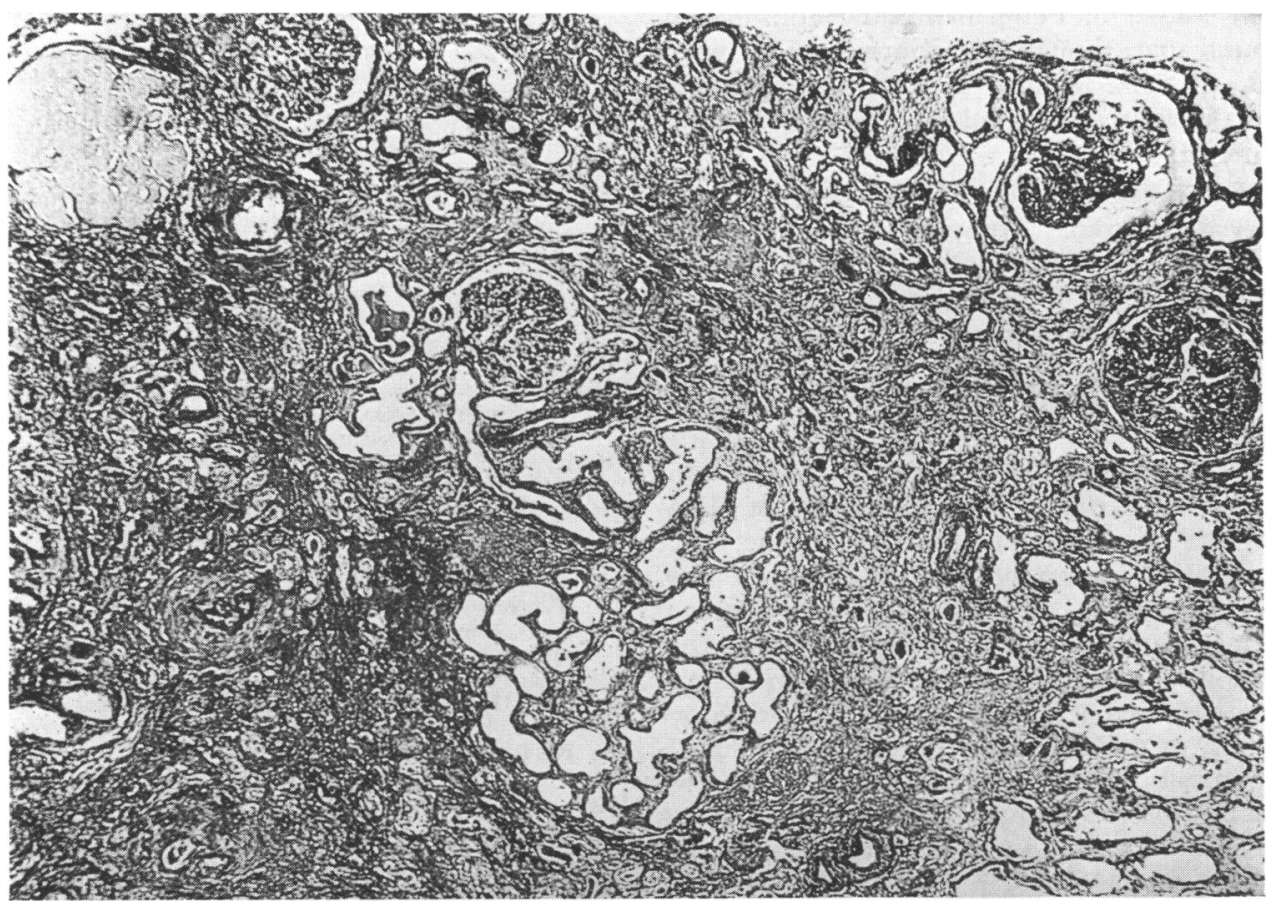

FIG. 4.-Case 1. Kidney showing 'thyroid-like' picture due to dilatation of the tubules. (Trichrome. $\times 45$.) 
light on certain aspects of the condition. Though the syndrome can present with less typical symptoms such as oedema and haematuria (Callis et al., 1970), a fairly uniform clinical picture is usual which can be ascribed to a progressive renal insufficiency and its repercussions on growth, acid-base balance, and calcium-phosphate metabolism (Royer et al., 1962, 1967a; Royer, Habib, and Leclerc, 1967b; Royer, Habib, and Mathieu, 1967c; Roget et al., 1965). Royer et al. (1967c) have drawn attention to the resemblance of some of these symptoms to those observed in experimental reduction of the nephron population. Calculation of the renal size and the glomerular surface at different intervals suggests that in oligonephronia normal growth of the existing nephrons occurs in the initial stages but that growth is finally inhibited by progressive sclerosis (Callis et al., 1970). It has further been shown that the actual glomerular filtration rate, not corrected for body surface, remains at a stable level for years (Royer et al., 1967c). Since the degree of renal hypoplasia varies from one patient to another, the time of appearance of the first symptoms and the severity of the symptomatology also varies, as illustrated by the histories of our four patients. Some of the biochemical findings are probably to be explained by a glomerulotubular imbalance, suggested by the microdissection studies of Fetterman and Habib (1969). They found that, though all nephrons were hypertrophied, the size of the proximal tubules was disproportionately increased relative to that of the glomeruli.

One of the diagnostic elements is said to be the symmetry of the renal hypoplasia; for this reason the diagnosis of oligonephronia was initially rejected in Case 1. It appeared, however, that in the large series of Royer et al. (1967a), one patient with oligonephronia had also an aplasia of the other kidney: the authors considered this as an associated congenital anomaly. Besides the fact that the designation 'bilateral oligonephronia' seems confusing in cases like these, the association of oligonephronia of one kidney and aplasia of the other kidney could be important from the point of view of the pathogenesis. Though nothing is known with certainty, the most logical explanation is that a limitation of the embryonic formation of the nephrons occurred with subsequent compensatory hypertrophy of the existing nephrons. The simultaneous aplasia of the other kidney in these two cases could then eventually be explained as an extreme inhibition of the renal formation on that side.

Associated congenital anomalies apparently are exceptional in oligonephronia. Therefore, the fact that in Case 4 the oligonephronia was part of a complex syndrome of multiple congenital anomalies was surprising. In one of the patient's sisters with the same syndrome of multiple anomalies, the renal anomaly consisted of a simple hypoplasia. The question arises whether there is a relation with the oligonephronia, or whether this is coincidental.

The histological picture of the kidney in oligonephronia is characterized by a decreased number of nephrons and by hypertrophy of all the elements of these nephrons. Dysplastic lesions are never seen. Depending on the stage of the disease, progressive hyalinization and fibrosis of the glomeruli, atrophy and dilatation of the tubules, and changes of the interstitium and the blood vessels occur; different stages may be seen simultaneously. Oligonephronia is thus readily distinguished from other conditions with similar clinical manifestations such as familial nephronophthisis, other forms of total or partial renal hypoplasia with or without dysplasia, and secondary renal atrophy (Bernstein, 1968; Bernstein and Meyer, 1964).

\section{REFERENCES}

Bernstein, J. (1968). Developmental abnormalities of the renal parenchyma: renal hypoplasia and dysplasia. Pathology Annual, 3, 213.

Bernstein, J., and Meyer, R. (1964). Some speculations on the nature and significance of developmentally small kidneys (renal hypoplasia). Nephron, 1, 137.

Callis, L., Castello, F., Vidal, M. T., and de Fortuny, G. (1970). Hypoplasie rénale avec oligonéphronie. Archives Françaises de Pédiatrie, 27, 267.

Fetterman, G. H., and Habib, R. (1969). Congenital bilateral oligonephronic renal hypoplasia with hypertrophy of nephrons (oligoméganéphronie). Studies by microdissection. American fournal of Clinical Pathology, 52, 199.

Friedenberg, M. J., Walz, B. J., McAlister, W. H., Locksmith, J. P., and Gallagher, T. L. (1965). Roentgen size of normal kidneys. Radiology, 84, 1022.

Roget, J., Beauduing, A., Couderc, P., and Lagier, A. (1965). Un cas d'insuffisance rénale chronique à début précoce avec rachitisme sévére. Hypoplasie rénale bilatérale oligonéphronique. Pédiatrie, 20, 969.

Royer, P., Habib, R., Mathieu, H., and Courtecuisse, V. (1962). L'hypoplasie rénale bilatérale congénitale avec réduction du nombre et hypertrophie des néphrons chez l'enfant. Annales de Pédiatrie, $8,133$.

Royer, P., Habib, R., Courtecuisse, V., and Leclerc, F. (1967a) L'hypoplasie rénale bilatérale avec oligonéphronie. Archives Françaises de Pédiatrie, 24, 249.

Royer, P., Habib, R., and Leclerc, F. (1967b). L'hypoplasie rénale bilatérale avec oligoméganéphronie. In Proceedings of the 3rd International Congress of Nephrology, Washington 1966, Vol. II, p. 251. Karger, Basle and New York.

Royer, P., Habib, R., and Mathieu, H. (1967c). Nephrologie im Kindesalter. Thieme, Stuttgart.

Correspondence to Dr. K. Van Acker, Kindergeneeskunde Rÿksuniversiteit, De Pintelaan 115, Gent, Belgium. 